\title{
Prehospital Plasma Transfusion: What Does the Literature Show?
}

\author{
Bryon P. Jackson ${ }^{a}$ Jason L. Sperry ${ }^{b}$ Mark H. Yazer ${ }^{c}$ \\ aDepartment of Pathology, University of Maryland School of Medicine, Baltimore, MD, USA; ${ }^{b}$ Department of \\ Surgery, University of Pittsburgh School of Medicine, Pittsburgh, PA, USA; 'Department of Pathology, University of \\ Pittsburgh School of Medicine, Pittsburgh, PA, USA
}

\author{
Keywords \\ Prehospital · Plasma · Transfusion
}

\section{Abstract}

Background: Early initiation of blood products transfusion after injury has been associated with improved patient outcomes following traumatic injury. The ability to transfuse patients' plasma in the prehospital setting provides a prime opportunity to begin resuscitation with blood products earlier and with a more balanced plasma:RBC ratio than what has traditionally been done. Published studies on the use of prehospital plasma show a complex relationship between its use and improved survival. Summary: Examination of the literature shows that there may be a mortality benefit from the use of prehospital plasma, but that it may be limited to certain subgroups of trauma patients. The likelihood of realizing these survival benefits appears to be predicted by several factors including the type of injury, length of transport time, presence of traumatic brain injury, and total number of blood products transfused, whether the patient required only a few products or a massive transfusion. When taken as a whole the evidence appears to show that prehospital plasma may have a mortality benefit that is most clearly demonstrated in patients with blunt injuries, moderate transfusion requirements, traumatic brain injury, and/or transport time greater than $20 \mathrm{~min}$, as well as those who demonstrate a certain cytokine expression profile. Key Messages: The evidence suggests that a targeted use of prehospital plasma will most likely maximize the benefits from the use of this limited resource. It is also possible that prehospital plasma may best be provided through whole blood as survival benefits were greatest in patients who received both prehospital plasma and RBCs.

(c) 2021 The Author(s).

Published by S. Karger AG, Basel

\section{Introduction}

Death due to hemorrhage is a leading cause of mortality worldwide and results in a significant loss of life years [1]. Over the course of time, various resuscitation strategies have been used to address this clinical problem. In the recent past, a resuscitation model based on crystalloid as the main fluid dominated clinical practice. Improved understanding of the risk of crystalloid and the need for early inclusion of blood products has resulted in newer resuscitation practices [2], specifically, Damage Control Resuscitation (DCR), which emphasizes the early inclusion of blood products and focuses on providing the patient with balanced resuscitation in ratios that approximate $1: 1$ [3].

In addition to the early inclusion of blood products in the resuscitation of injured patients, the overall time to initiation of transfusion has been shown as a critical factor in reducing mortality $[4,5]$. The ability to transfuse patients in the prehospital setting provides a prime opportunity to begin resuscitation with blood products earlier and with a more balanced plasma:RBC ratio than what has traditionally been done. It would seem logical that the initiation of blood product transfusion in the prehospital setting would improve patient outcomes. How-

\section{karger@karger.com} www.karger.com/tmh

Karger"

BOPEN ACCESS
(C) 2021 The Author(s)

Published by S. Karger AG, Basel

This is an Open Access article licensed under the Creative Common Attribution-NonCommercial-4.0 International License (CC BY-NC) (http://www.karger.com/Services/OpenAccessLicense), applicable to the online version of the article only. Usage and distribution for commercial purposes requires written permission.
Correspondence to:

Bryon P. Jackson, bryonpjackson@gmail.com 


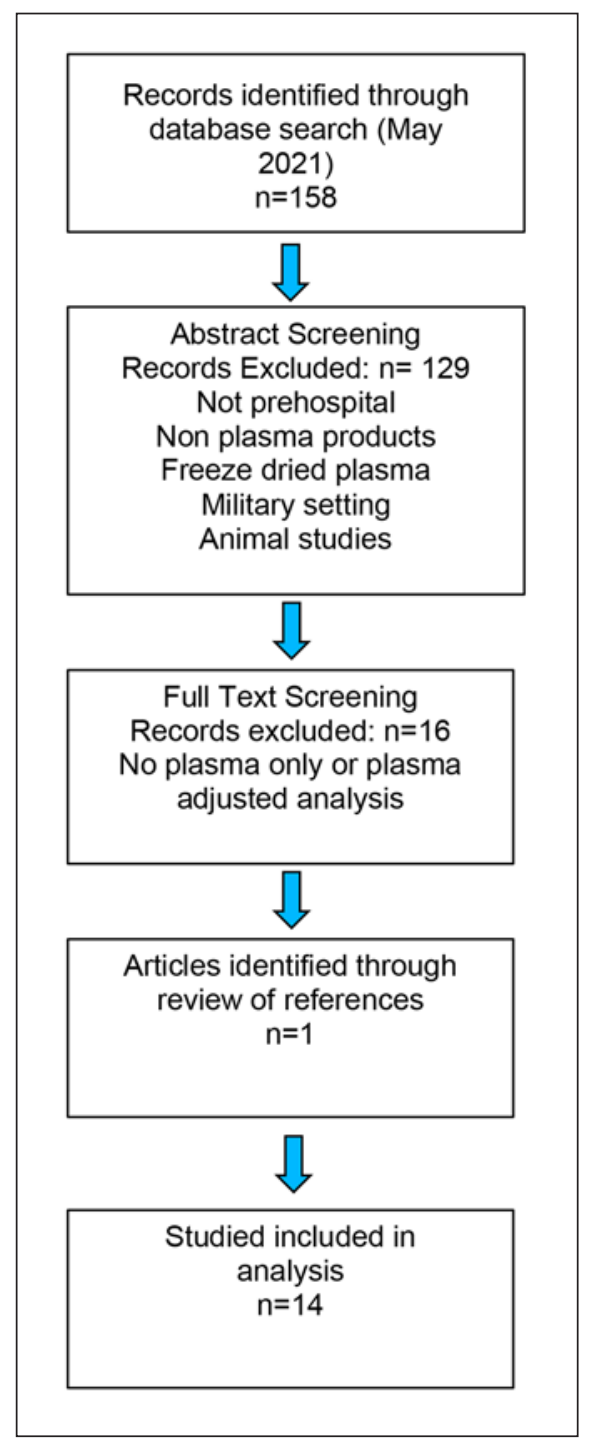

Fig. 1. Identification, review, and selection of included studies.

ever, research on the outcomes and benefits of prehospital plasma transfusion (PHP) in particular has shown that the answer may be much more complex. Studies to date have shown that there are numerous factors that can impact the likelihood that PHP will improve the patient's outcome. In fact, the two largest randomized trials conducted on this topic showed conflicting results $[6,7]$.

If PHP is to be widely adopted, it is critical to understand and identify the factors that affect its effectiveness in order to target the patients and clinical situations where the benefits of PHP will likely be maximized. Additionally, it is important to understand the outcomes and metrics that should be used to classify the benefits of PHP. As the effects of prehospital plasma are likely multifactorial, understanding its impact will require examination of more nuanced outcomes beyond simple measurements of mortality. To that end, this review aims to analyze the current evidence for the use of PHP and identify further opportunities for research to provide a level of clarity that can improve clinical decision-making. This review will not address the use of freeze-dried plasma, plasma used in military settings, and papers that examined prehospital transfusion of other blood products without an adjusted analysis for plasma transfusion.

\section{Methods}

Articles included in this analysis were identified through a PubMed search using medial subject headings: prehospital, plas$\mathrm{ma}$, and transfusion. Studies were included if they reported on transfusion of plasma used in a prehospital civilian setting with the use of plasma as the primary variable and measured safety, feasibility, or a direct clinical outcome. Non-comparative studies, review articles, or articles not available in English were excluded from analysis. Adherence to the inclusion criteria was determined by review of abstracts and/or full text of the articles identified. Additional articles were identified and included through review of the references of previously included articles if they met all inclusion criteria (Fig. 1).

\section{Results}

The initial search yielded 158 results. One hundred twenty-nine articles were excluded based on review of the abstract. These studies were excluded because the plasma transfusions were not administered in the prehospital setting, were focused on prehospital transfusion of nonplasma products, used freeze-dried plasma, were performed in a military setting, were animal studies, were limited to description of study design, contained only in vitro studies, or presented methods to implement a prehospital transfusion program without actual transfused patient data. Twenty-nine articles underwent full text review. Sixteen articles were excluded based on full text review for presenting studies of prehospital transfusion of plasma and RBCs without an adjusted analysis for the transfused plasma. The remaining 13 articles were included in the review. Review of the references list of the included articles yielded one additional article that met the inclusion criteria and was therefore included in this analysis for a total of 14 articles [6-19].

Among the included studies were two randomized controlled trials $[6,7]$, a single-center prospective observational study [18], and one single-center retrospective observational study [19] (Table 1). The remaining studies were secondary analyses of data from either the Prehospital Plasma during Air Medical Transport in Trauma Patients at Risk for Hemorrhagic Shock (PAMPer) trial [8$12,14,17]$ or a combined data set from COMBAT and PAMPer $[13,15,16]$. All studies included analysis of a cohort of adult trauma patients who were transfused with 
Table 1. Study design

\begin{tabular}{|c|c|c|c|}
\hline & Study type/data source & Intervention & Primary outcome \\
\hline \multicolumn{4}{|l|}{ Primary study } \\
\hline Kim 2012 & Retrospective observational & Two units of thawed plasma & Hemostatic function measured by INR \\
\hline Henriksen 2016 & Prospective observational & Two units of thawed plasma & Hemostatic function measured by rTEG \\
\hline Moore 2018 & Randomized controlled trial & $\begin{array}{l}500 \mathrm{~mL} \text { of plasma thawed } \\
\text { immediately prior to use }\end{array}$ & 24-h and 28-day mortality \\
\hline Sperry 2018 & Randomized controlled trial & Two units of thawed plasma & 24-h and 30-day mortality \\
\hline \multicolumn{4}{|l|}{ Secondary analysis } \\
\hline Anto 2020 & PAMPer & & Mortality in patient requiring an MT vs. no MT \\
\hline Gruen JAMA 2020 & PAMPer & & Mortality with TBI vs. no TBI \\
\hline Gruen JTACS 2020 & PAMPer & & Unexpected survival \\
\hline Gruen JCI 2020 & PAMPer & & Expression of biomarkers \\
\hline Guyette 2021 & PAMPer & & $\begin{array}{l}\text { Mortality based on products transfused } \\
\text { (RBCs + plasma, plasma, } R B C s \text {, or crystalloid) }\end{array}$ \\
\hline Moore 2020 & PAMPer plus COMBAT & & Risk of hypocalcemia \\
\hline Pusateri 2020 & PAMPer plus COMBAT & & $\begin{array}{l}\text { Mortality based on transport time greater or } \\
\text { less than } 20 \mathrm{~min}\end{array}$ \\
\hline Reitz 2020 & PAMPer plus COMBAT & & Mortality: blunt vs. penetrating injury \\
\hline Reitz 2021 & PAMPer & & Mortality based on age of plasma \\
\hline Sim 2020 & PAMPer & & $\begin{array}{l}\text { Mortality based on achievement if various } \\
\text { CAT }\end{array}$ \\
\hline
\end{tabular}

MT, massive transfusion; CAT, critical administration threshold.

either prehospital plasma or the center's standard of care treatment as the control group. Adult was defined as 18 and older in all studies except in Henriksen et al. [18], where it was defined as greater than 16 years of age. Henrikesen et al. [18] included all trauma patients that were transfused either in the prehospital setting or within $6 \mathrm{~h}$ of admission. Kim et al. [19] included all patients that met institutional guidelines for activation of a massive transfusion event. PAMPer and COMBAT included trauma patients with systolic blood pressure (SBP) $<70 \mathrm{~mm} \mathrm{Hg}$ or SBP between 70 and $90 \mathrm{~mm} \mathrm{Hg}$ and heart rate greater than $108[6,7]$.

The PAMPer study (and all secondary analyses) and Kim et al. [19] both used plasma that had been thawed in the blood bank, while COMBAT provided plasma that was thawed immediately prior to transfusion while the patient was in the ambulance $[6-12,14,19]$. Henriksen et al. [18] did not specify when the plasma was thawed. All studies used group $\mathrm{AB}$ or A plasma with PAMPer indicating that the group A plasma featured a low titer of anti-B. Across all studies, patients could receive a maximum of two units or $500 \mathrm{~mL}$ of plasma in the prehospital setting. In addition to plasma, prehospital RBCs and crystalloid could also have been transfused if they were part of the center's standard of care to the patients who were in the standard of care arm in all studies except for the COMBAT trial where the control arm received only crystalloid. COMBAT specified the use of normal saline, while the other studies did not specify the type of crystalloid used
$[6,7,18,19]$. Tranexamic acid was used in the COMBAT study, but the frequency of its use was not significantly different between the two groups, and no specific analysis was performed regarding its use. The other primary studies did not provide information on the use of TXA $[6,7$, $18,19]$.

The total number of subjects included in the primary studies ranged from 59 to 501 . The number of subjects in the combined PAMPer and COMBAT analyses ranged from 160 to 626 and ranged from 166 to 501 in the secondary analyses of the PAMPer study. Patients were transported by air medical transport in two of the primary analyses $[7,19]$ and in all of the secondary analyses of the PAMPer study $[8-12,14,17]$. In the COMBAT trial patients were transported via ground medical transport. Patients in Henriksen et al. [18] and the combined PAMPer/COMBAT analyses $[13,15,16]$ were transported via either ground or air medical transport as specified by the trial in which they were enrolled. Median transport times from the scene of the accident to the hospital were reported in all primary analyses with the exception of Henriksen et al. [18]. The median transport time was not significantly different between the prehospital plasma group and control in those studies where these data were reported; however, there was a major difference between patients in the COMBAT and PAMPer trials (Table 2). The proportion of patients with blunt injuries was higher in the PAMPer study. Patients in the PAMPer trial also tended to be older and have lower injury severity scores 
Table 2. Primary analysis studies

\begin{tabular}{|c|c|c|c|c|c|c|c|c|}
\hline & \multicolumn{2}{|c|}{ PAMPer } & \multicolumn{2}{|c|}{ СOMBAT } & \multicolumn{2}{|l|}{ Henriksen } & \multicolumn{2}{|l|}{ Kim } \\
\hline & FFP & $\begin{array}{l}\text { Standard } \\
\text { care }\end{array}$ & FFP & $\begin{array}{l}\text { Standard } \\
\text { care }\end{array}$ & FFP & $\begin{array}{l}\text { Standard } \\
\text { care }\end{array}$ & FFP & $\begin{array}{l}\text { Standard } \\
\text { care }\end{array}$ \\
\hline \multicolumn{9}{|l|}{ Patient characteristics } \\
\hline Number & 230 & 271 & 65 & 60 & 75 & 182 & 9 & 50 \\
\hline Age (median) & 44 & 46 & 33 & 32.5 & 34 & 39 & 54 & 41 \\
\hline Injury severity score & 22 & 21 & 27 & NR & 29 & 26 & 27 & 23 \\
\hline Blunt injury, $\%$ & 81.3 & 83.4 & 46 & 53 & 54.7 & 74.7 & 67 & 82 \\
\hline Transport type & Air & Air & Ground & Ground & Air & Air & Air & Air \\
\hline Transport time & 42 & 40 & 19 & 16 & NR & NR & 40 & 39 \\
\hline \multicolumn{9}{|l|}{ Primary outcomes } \\
\hline \multicolumn{9}{|l|}{ Mortality, \% } \\
\hline 24-hour & 13.9 & 22.1 & 12 & 10 & 16 & 10.4 & 44 & 10 \\
\hline 28/20-day & 23.2 & 33 & 15 & 10 & & & & \\
\hline rTEG MA, mm & & & & & 62.55 (2 units) & 58.73 & & \\
\hline Change in INR & & & & & & & 0.9 & 0.2 \\
\hline
\end{tabular}

as well as more frequently required massive transfusion than those in COMBAT $[6,7]$.

Mortality (30- or 28-day, respectively) was the primary outcome for the PAMPer and COMBAT studies [6, 7]. The primary outcome in Henriksen et al. [18] and Kim et al. [19] was hemostatic function assessed by thromboelastography (TEG) and INR, respectively. Other non-mortality outcomes were measured as the primary outcome in Moore et al. [15] (risk of hypocalcemia) and Gruen et al. [11] (expression of biomarkers for inflammation and endothelial function/damage) and are shown in Table 1.

Among studies with mortality as the primary outcome, PAMPer demonstrated lower 30-day mortality in the group of patients who received PHP compared to those in the standard of care arm $(23.2 \%$ vs. $33.0 \%$, respectively, $p=0.02$ ) [7], while the COMBAT trial showed no significant difference in mortality between the plasma versus standard of care recipients (15\% vs. $10 \%$, respectively, $p=0.37$ ) [6] (Table 2). In a combined analysis of PAMPer and COMBAT, Reitz et al. [13] showed a mortality benefit in patients with blunt injury (32\% lower independent hazard for 28-day mortality, HR 0.68, 95\% CI $0.47-0.96, p=0.03)$, but not in those with penetrating injuries (HR 1.16, 95\% CI 0.4-3.1, $p=0.78$ ). In a separate combined analysis, Pusateri et al. [16] showed a mortality benefit in prehospital patients who received plasma and who had a transport time greater than 20 min compared to those with a similar transport time but without PHP (HR 0.78, 95\% CI 0.40-1.51, $p=0.46$ ) where prehospital time is defined as arrival on scene to arrival in ED.

In secondary analysis of PAMPer data, Gruen et al. [9] showed PHP was associated with improved survival in patients with traumatic brain injury (TBI) (HR 0.56, 95\% CI 0.35-0.91) and polytrauma (HR 0.50, 95\% CI 0.28-0.89) compared to those without those conditions. In another secondary analysis of the PAMPer study, Sim et al. [17] showed that mortality varied based on the definition of massive transfusion applied to the study cohort with overall study mortality rates of $13.1 \%, 17.4 \%$, and $10.0 \%$ based on CAT1hr ( $\geq 3$ units per hour in the first hour), CAT4hr ( $\geq 3$ units per hour in any of the first $4 \mathrm{~h}$ ), and historic massive transfusion definitions ( $\geq 10$ units of RBCs in $24 \mathrm{~h}$ ), respectively. Anto et al. [8] showed lower mortality in patients who required a massive transfusion, defined as receipt of more than $10 \mathrm{RBC}$ units in $24 \mathrm{~h}$, compared to those who did not require a massive transfusion $(42 \%$ vs. $26 \%$, respectively, $p=0.001$ ). Reitz et al. [12] demonstrated that the age of the thawed plasma, i.e., the number of days post-thaw before it was transfused, did not impact mortality or biomarker expression in a secondary analysis of PAMPer data. Guyette et al. [14] demonstrated that the greatest mortality reduction was seen in patients who received both plasma and RBCs in the prehospital setting (HR 0.38, 95\% CI 0.26-0.55, $p<0.001$ ). This analysis also showed that PHP alone (HR 0.57, 95\% CI 0.36-0.91, $p<$ 0.001 ) produced the second lowest mortality rate followed by the prehospital transfusion of RBCs alone (HR 0.68, $95 \%$ CI $0.49-0.95, p<0.001)$. In this analysis, the patients who received only saline had the highest 30 -day mortality (HR 1.65, 95\% CI 1.17-2.32, $p<0.001$ ) [14].

Henriksen et al. [18] did not show a difference in inhospital mortality between patients who received PHP and those treated with standard of care $(26.7 \%$ vs. $20.9 \%$, $p=0.313$ ). The study did show that when adjusted for prehospital RBC transfusions, the administration of prehospital plasma was associated with a significantly higher rTEG MA (58.73 vs. $62.55 \mathrm{~mm}, p=0.012$ ) at the time of admission compared to those treated with standard care. 
Kim et al. [19] demonstrated a greater correction of coagulopathy as measured by the INR in the patients who received $\mathrm{PHP}$ at the initiation of transport versus arrival at trauma center, which was not statistically significant ( 0.9 vs. $0.2, p=0.078)$. This analysis also showed that the transfusion of prehospital plasma was associated with a plasma:RBC ratio that more closely approximated 1:1 at $30 \mathrm{~min}$ and at $24 \mathrm{~h}$ after admission compared to those treated with standard of care [18].

In a combined analysis of PAMPer and COMBAT data, Moore et al. [15] showed an increased risk of hypocalcemia in patients who received prehospital plasma compared to those who did not ( $53 \%$ vs. $36 \%$, adjusted relative risk $1.48,95 \%$ CI $1.03-2.12, p=0.03$ ). This study also showed that after adjusting for confounders, severe hypocalcemia was associated with decreased survival and the need for massive transfusion [15]. In an analysis of the most severely injured patients, Gruen et al. [11] showed that prehospital plasma was associated with an increase in adiponectin, IL-1 $\beta$, IL-17A, IL-23, and IL-17E upon admission, and a reduction in syndecan-1, TM, VEGF, IL-6, IP-10, MCP-1, and TNF- $\alpha$, and an increase in IL-33, IL-21, IL-23, and IL-17E $24 \mathrm{~h}$ later.

\section{Discussion}

On the surface, it would appear that the ability to transfuse blood products, specifically plasma, in the prehospital setting would have obvious clinical benefits in the treatment of trauma patients. However, examination of the published studies on the use of prehospital plasma show a more complex relationship between its use and improved survival. Examination of the literature shows that there may be a mortality benefit, but that it may be limited to certain subgroups of trauma patients. The likelihood of realizing these survival benefits appears to be predicted by several factors including the type of injury, length of transport time, presence of TBI, and total number of blood products transfused, whether the patient required only a few products or a massive transfusion. The practical application of the existing data must be evaluated in the context of study size and design and the subsection of trauma patients included in the respective studies.

To understand the circumstances where PHP is most likely to have an impact it is important to understand the mechanisms by which it is expected to impact mortality. Among the theorized mechanisms of action for PHP are treatment of coagulopathy [20], changes in the inflammatory response to injury [21], and repair of the endothelial lining [22].

It is important to keep in mind that of the 14 studies analyzed herein, only 4 presented primary data and the remainder performed secondary analyses of the primary data and must be viewed with the understanding that the original study was not always designed to facilitate the detection of a difference in these secondary analyses. This is the nature of performing a secondary analysis. The overwhelming number of subjects and the only randomized controlled trial data come from the COMBAT and PAMPer trials. These two studies present conflicting conclusions with PAMPer showing a mortality benefit from PHP while no difference was seen in COMBAT. There are several factors that may explain why these two trials with similar inclusion criteria and treatment protocols reached different conclusions. One possible explanation for the difference is chance and the lower power of the COMBAT study due to its smaller sample size (125 patients in COMBAT vs. 501 in PAMPer). There were also differences in patients' demographics with PAMPer patients tending to be older, more likely to be female, more likely to have a blunt injury, have a lower injury severity score and longer median transport times compared to the patients in COMBAT. Given the shorter median transport time in COMBAT (19 min vs. $41 \mathrm{~min}$ in PAMPer), it is possible that the difference in time to initial transfusion between the PHP and control groups was not sufficient to show a mortality benefit in COMBAT as the control patients did not have to wait for long to receive their plasma when they arrived at the hospital. It should also be noted that there was a difference in the relative amount of crystalloid administered in the standard of care and PHP groups between the studies with the standard care group in PAMPer receiving an average of $400 \mathrm{~mL}$ more crystalloid compared to the standard of care group in COMBAT who received only 100 $\mathrm{mL}$ more when compared to their respective PHP groups. Given the known association of higher volumes of crystalloid with worse outcomes this could also have contributed to the difference in observed results [2].

Taken as a whole these data support a morality benefit of PHP that is most clearly seen in trauma patients with certain characteristics. When the COMBAT and PAMPer data sets were combined, the analysis showed the mortality benefit associated with PHP was seen primarily in patients with blunt injuries and not those with penetrating injuries [13]. The difference in outcomes observed between these two groups may have resulted from the sample size of patients with penetrating injuries being too small to show a potential mortality difference. It is also possible that if the benefit of PHP is related more to the proposed inflammatory benefits, particularly endothelial lining protection, it would likely provide greater benefit in a patient with a multisystem blunt injury pattern [23-25]. Alternatively, the use of PHP may have counteracted the benefits of permissive hypotension that have been shown in patients with penetrating injuries $[2,26]$. There is also the potential of survivor bias resulting from patients with the earliest deaths more likely to have penetrating injuries and therefore unable to be enrolled before death. 
The literature demonstrates that transport time appears to be an important factor in identifying which patients will benefit from PHP. The combined analysis of PAMPer and COMBAT showed that transport times longer than 20 min were associated with a mortality benefit from receipt of PHP [16]. The selection of the 20-min time point was supported by a sensitivity analysis that showed a change in response to PHP was evident for prehospital times longer than $20 \min (p=0.003$ vs. $p=0.007$ for 17 $\min , p=0.006$ for $22 \mathrm{~min}, p=0.01$ for $25 \mathrm{~min}$, and $p=0.02$ for $30 \mathrm{~min}$ ) [16]. This distinction could potentially explain the results of the initial analyses whereby COMBAT patients had shorter median transport times and no mortality benefit was observed. With shorter transport times it is possible that in-hospital transfusion was not delayed to a degree that would allow for observation of the negative effect resulting from delayed time to transfusion as was apparent in the control arm patients in the PAMPer study [27]. This difference also persisted when adjusted for injury severity which was higher in the PAMPer cohort.

The presence or absence of TBI was associated with a difference in the observed benefit of PHP. In patients with TBI, early interventions that reduce hypoxia, hypotension, and hypocarbia have been shown to improve outcomes [28-31]. There was also a pair of observational studies that suggested a benefit of early in-hospital plasma transfusion in patients with TBI $[32,33]$. It would seem logical that this benefit from early in-hospital transfusion could be extrapolatable to the benefit observed from PHP transfusion in injured patients with TBI. In line with this expectation, a secondary analysis of the PAMPer data demonstrated improved survival among patients with TBI (GCS less than 8) and those with polytrauma [9]. The association of benefit in TBI with polytrauma may also suggest that other systemic benefits may contribute to the observed difference in mortality.

When considering the effect that receipt of a massive transfusion might have on the benefit of PHP receipt, the definition of massive transfusion used may be an important factor to consider. This definition was not the same for all studies and depended on the respective inclusion criteria for each study. The results suggest that the benefit of PHP is associated with the overall volume of transfusion. Separate analyses demonstrated that the mortality benefit of PHP was strongest in patients with moderate transfusion needs defined by sub-MT red cell requirements (4-7 units in $24 \mathrm{~h}$ ) [8] and adjustment of the critical administration threshold (CAT) ( $\geq 3$ units of RBCs transfused in any of the first $4 \mathrm{~h}$ ) [17]. The idea that patients with moderate transfusion requirements would benefit most from PHP is both logical and in line with existing data. It may be the case that patients with small transfusion requirements either died soon after admission without time to reach the CAT or were not severely injured enough to require enough blood prod- ucts to meet CAT at any time. Alternatively, patients who reached the CAT at $1 \mathrm{~h}$ would likely be more severely injured and have a higher risk of death. This logic is supported by the finding in Anto et al. [8] where a survival benefit in massively transfused patients (greater than 10 RBCs in $24 \mathrm{~h}$ ) or patients who received fewer than 4 units of RBCs in $24 \mathrm{~h}$ was not found. This suggests there may be an optimal patient population with injuries that meet a CAT while not requiring a massive transfusion that would benefit from PHP. PHP may not be optimal for patients with a low chance of survival based on high early transfusion requirements. There is also the question of what impact the PHP had on transfusion requirements. It is unclear if the PHP prevented patients from requiring a massive transfusion who would have if not for the prehospital intervention. Predicting the risk of massive transfusion may be a better assessment tool than who actually received a massive transfusion. It is also worth considering more broadly if the historic definitions of massive transfusion are best suited for this type of analysis or if there should be a focus on smaller transfusion volumes over shorter periods of time [34]. In either case, the challenge will still be predicting which patients are likely to fall into the moderate transfusion group particularly in the prehospital environment when decisions about administering PHP are being made.

Another important consideration is the inclusion of RBCs and crystalloid in the prehospital regimen. The evidence against copious crystalloid resuscitation has been well established $[3,35]$, but it is less clear if RBCs or plasma alone would be superior to the other if only one product could be made available. The inclusion of RBCs as part of the standard of care at some of the PAMPer sites permitted a stratification and comparison of groups that received plasma + RBCs, plasma, RBCs, or crystalloid. The conclusion that the combination of plasma and RBCs provided the greatest survival benefit is logical given it provides a resuscitation that would more closely resemble what the patient is bleeding, and it is similar to whole blood $[36,37]$. Plasma alone was shown to be superior to RBCs alone and this would suggest that if only one blood product can be provided plasma should be given priority [14]. This data also points towards serious consideration for the use of whole blood as a way to provide both plasma and RBCs with the added benefit of platelets in one product.

Moving beyond mortality, the literature also provides some insight into some additional clinical considerations. One of these is the observation of hypocalcemia in patients receiving PHP in a combined analysis of COMBAT and PAMPer [15]. Hypocalcemia has been shown to be a sequelae of transfusion and moreover severe hypocalcemia has been associated with increased risk of death in massively transfused patients [38-40]. This relationship may be relevant as it informs our interpretation of the current data and also to how to practically address the is- 
sue moving forward. It is important to consider that the hypocalcemia associated with the use of PHP may have dampened the observed benefit of PHP across all included studies. It also suggests that calcium supplementation in massively transfused patients is a topic that deserves further consideration and additional research.

In animal and in vitro studies, the beneficial effects of plasma on endothelial permeability and vascular stability have been shown to be independent of hemostasis and also to decrease with storage [41]. The results of Reitz et al. [12] suggest that while the age of the thawed plasma transfused showed no impact on mortality there were some changes in inflammatory and endothelial lining biomarker expression in severely injured patients. Specifically, old (day 2-5) plasma was associated with increased adjusted inflammatory and decreased adjusted endothelial biomarkers. More extensive analysis of biomarker expression showed PHP patients had lower levels of proinflammatory meditators and markers of endothelial lining damage regardless of plasma storage duration [12]. This suggests the effects of PHP may impact the underlying physiology of trauma beyond simply the replacement of clotting factors.

While the underlying impact of plasma is important to understand there are also clinical markers that are impacted by the use of PHP. In the two studies with coagulation parameters as the primary outcome $[18,19]$ there was a demonstrated improvement in INR correction or TEG MA in patients who received PHP. Henriksen et al. [18] was limited as the two groups had notable differences in $\mathrm{pH}$ and platelet count that may have independently predicted reduced hemostatic competence and future analysis should consider adjusting for $\mathrm{pH}$ and platelet count. It should be noted that the difference in INR correction observed in Kim et al. [19] was not significant and was based on a PHP sample size of 9 patients. Both functional and non-functional measures were studied and showed improvement but were not without significant limitations in study design. Henriksen et al. [18] also suggested a role of PHP in early correction of acidosis which could further mitigate the traumatic coagulopathy.

The plasma product and dose are factors that could potentially impact the effect observed in the use of PHP. In the studies examined, the dose of plasma was limited to approximately two units or $500 \mathrm{~mL}$. The normal recommended dose of plasma to correct a coagulopathy has been suggested to be $10-15 \mathrm{~mL} / \mathrm{kg}$. In a study that measured clotting factors before and after receipt of four units of FFP there was an increase in clotting factor levels that ranged from 9 to $14 \%$ [42]. Given the doses used in the included studies it is likely that clotting factors were increased by less than $10 \%$. This supports the idea that the impact of PHP where observed was not strictly a function of correcting a coagulopathy.

\section{Conclusion}

When taken as a whole the evidence appears to show that the PHP may have a mortality benefit that is most clearly demonstrated in patients with blunt injuries, moderate transfusion requirements, TBI, and/or transport time greater than $20 \mathrm{~min}$, as well as those who demonstrate a certain cytokine expression profile. These conclusions were based heavily on the evidence provided in the data from two randomized controlled trials. What remains to be clearly elucidated is the precise mechanism by which PHP produces its benefit. Given the variety of patients and doses used it appears likely that the mechanism of benefit is multifactorial and may in fact be different in different subpopulations of trauma patients. There is a need for more studies focused on identifying the mechanisms of plasma's activity and correlations made with various trauma patient populations. The evidence suggests that a targeted use of PHP will most likely maximize the benefits from the use of this limited resource. It is also possible that PHP may best be provided through whole blood as survival benefits were greatest in patients who received both PHP and RBCs.

\section{Conflict of Interest Statement}

The authors have no conflicts of interest to declare.

\section{Funding Sources}

Drs Sperry and Yazer were the PI and a co-investigator, respectively, on the PAMPer study, funded by the U.S. Army Medical Research and Materiel Command; PAMPer, ClinicalTrials.gov number, NCT01818427.

\section{Author Contributions}

B.P.J., J.L.S., and M.H.Y. contributed to the design and implementation of the research, to the analysis of the results, and to the writing of the manuscript.

References

1 Cannon JW. Hemorrhagic Shock. N Engl J Med. 2018;378(4):370-9.

2 Bickell WH, Wall MJ, Pepe PE, Martin RR, Ginger VF, Allen MK, et al. Immediate versus delayed fluid resuscitation for hypotensive patients with penetrating torso injuries. $\mathrm{N}$ Engl J Med. 1994;331(17):1105-9.

3 Butler FK, Holcomb JB, Schreiber MA, Kotwal RS, Jenkins DA, Champion HR, et al. Fluid Resuscitation for Hemorrhagic Shock in Tactical Combat Casualty Care: TCCC Guidelines Change 14-01--2 June 2014. J Spec Oper Med. 2014;14(3):13-38. 
4 Holcomb JB, del Junco DJ, Fox EE, Wade CE, Cohen MJ, Schreiber MA, et al. The prospective, observational, multicenter, major trauma transfusion (PROMMTT) study: comparative effectiveness of a time-varying treatment with competing risks. JAMA Surg. 2013;148(2):127-36.

5 Zink KA, Sambasivan CN, Holcomb JB, Chisholm G, Schreiber MA. A high ratio of plasma and platelets to packed red blood cells in the first 6 hours of massive transfusion improves outcomes in a large multicenter study. Am J Surg. 2009;197(5):565-70; discussion 570.

6 Moore HB, Moore EE, Chapman MP, McVaney K, Bryskiewicz G, Blechar R, et al. Plasma-first resuscitation to treat haemorrhagic shock during emergency ground transportation in an urban area: a randomised trial. Lancet. 2018; 392(10144):283-91.

7 Sperry JL, Guyette FX, Brown JB, Yazer MH, Triulzi DJ, Early-Young BJ, et al. Prehospital Plasma during Air Medical Transport in Trauma Patients at Risk for Hemorrhagic Shock. N Engl J Med. 2018;379(4):315-26.

8 Anto VP, Guyette FX, Brown J, Daley B, Miller R, Harbrecht B, et al. Severity of hemorrhage and the survival benefit associated with plasma: Results from a randomized prehospital plasma trial. J Trauma Acute Care Surg. 2020;88(1):141-7.

9 Gruen DS, Guyette FX, Brown JB, Okonkwo DO, Puccio AM, Campwala IK, et al. Association of Prehospital Plasma With Survival in Patients With Traumatic Brain Injury: A Secondary Analysis of the PAMPer Cluster Randomized Clinical Trial. JAMA Netw Open. 2020; 3(10):e2016869.

10 Gruen DS, Guyette FX, Brown JB, Daley BJ, Miller RS, Harbrecht BG, et al. Characterization of unexpected survivors following a prehospital plasma randomized trial. J Trauma Acute Care Surg. 2020;89(5):908-14.

11 Gruen DS, Brown JB, Guyette FX, Vodovotz Y, Johansson PI, Stensballe J, et al. Prehospital plasma is associated with distinct biomarker expression following injury. JCI Insight. 2020; 5(8).

12 Reitz KM, Gruen DS, Guyette F, Brown JB, Yazer MH, Vodovotz Y, et al. Age of thawed plasma does not affect clinical outcomes or biomarker expression in patients receiving prehospital thawed plasma: a PAMPer secondary analysis. Trauma Surg Acute Care Open. 2021;6(1): e000648.

13 Reitz KM, Moore HB, Guyette FX, Sauaia A, Pusateri AE, Moore EE, et al. Prehospital plasma in injured patients is associated with survival principally in blunt injury: Results from two randomized prehospital plasma trials. J Trauma Acute Care Surg. 2020;88(1):33-41.

14 Guyette FX, Sperry JL, Peitzman AB, Billiar TR, Daley BJ, Miller RS, et al. Prehospital Blood Product and Crystalloid Resuscitation in the Severely Injured Patient: A Secondary Analysis of the Prehospital Air Medical Plasma Trial. Ann Surg. 2021;273(2):358-64.

15 Moore HB, Tessmer MT, Moore EE, Sperry JL, Cohen MJ, Chapman MP, et al. Forgot calcium? Admission ionized-calcium in two civilian randomized controlled trials of prehospital plasma for traumatic hemorrhagic shock. J Trauma Acute Care Surg. 2020;88(5):588-96.

16 Pusateri AE, Moore EE, Moore HB, Le TD, Guyette FX, Chapman MP, et al. Association of
Prehospital Plasma Transfusion With Survival in Trauma Patients With Hemorrhagic Shock When Transport Times Are Longer Than 20 Minutes: A Post Hoc Analysis of the PAMPer and COMBAT Clinical Trials. JAMA Surg. 2020;155(2):e195085.

17 Sim ES, Guyette FX, Brown JB, Daley BJ, Miller RS, Harbrecht BG, et al. Massive transfusion and the response to prehospital plasma: It is all in how you define it. J Trauma Acute Care Surg. 2020;89(1):43-50.

18 Henriksen HH, Rahbar E, Baer LA, Holcomb JB, Cotton BA, Steinmetz J, et al. Pre-hospital transfusion of plasma in hemorrhaging trauma patients independently improves hemostatic competence and acidosis. Scand J Trauma Resusc Emerg Med. 2016;24(1):145.

19 Kim BD, Zielinski MD, Jenkins DH, Schiller HJ, Berns KS, Zietlow SP. The effects of prehospital plasma on patients with injury: a prehospital plasma resuscitation. J Trauma Acute Care Surg. 2012;73(2 Suppl 1):S49-53.

20 Maegele M, Schöchl H, Menovsky T, Maréchal H, Marklund N, Buki A, et al. Coagulopathy and haemorrhagic progression in traumatic brain injury: advances in mechanisms, diagnosis, and management. Lancet Neurol. 2017;16(8):63047.

21 Pati S, Peng Z, Wataha K, Miyazawa B, Potter DR, Kozar RA. Lyophilized plasma attenuates vascular permeability, inflammation and lung injury in hemorrhagic shock. PLoS One. 2018; 13(2):e0192363.

22 Barelli S, Alberio L. The Role of Plasma Transfusion in Massive Bleeding: Protecting the Endothelial Glycocalyx?. Front Med (Lausanne). 2018;5:91.

23 Hofmann N, Zipperle J, Jafarmadar M, Ashmwe M, Keibl C, Penzenstadler C, et al. Experimental Models of Endotheliopathy: Impact of Shock Severity. Shock. 2018;49(5):564-71.

24 Wu F, Chipman A, Pati S, Miyasawa B, Corash L, Kozar RA. Resuscitative Strategies to Modulate the Endotheliopathy of Trauma: From Cell to Patient. Shock. 2020;53(5):575-84.

25 Johansson PI, Henriksen HH, Stensballe J, Gybel-Brask M, Cardenas JC, Baer LA, et al. Traumatic Endotheliopathy: A Prospective Observational Study of 424 Severely Injured Patients. Ann Surg. 2017;265(3):597-603.

26 Schreiber MA, Meier EN, Tisherman SA, Kerby JD, Newgard CD, Brasel K, et al. A controlled resuscitation strategy is feasible and safe in hypotensive trauma patients: results of a prospective randomized pilot trial. J Trauma Acute Care Surg. 2015;78(4):687-95; discussion 695-7.

27 Meyer DE, Vincent LE, Fox EE, O'Keeffe T, Inaba K, Bulger E, et al. Every minute counts: Time to delivery of initial massive transfusion cooler and its impact on mortality. J Trauma Acute Care Surg. 2017;83(1):19-24.

28 Spaite DW, Hu C, Bobrow BJ, Chikani V, Barnhart B, Gaither JB, et al. Association of Out-ofHospital Hypotension Depth and Duration With Traumatic Brain Injury Mortality. Ann Emerg Med. 2017;70(4):522-530.e1.

29 Spaite DW, Hu C, Bobrow BJ, Chikani V, Sherrill D, Barnhart B, et al. Mortality and Prehospital Blood Pressure in Patients With Major Traumatic Brain Injury: Implications for the Hypotension Threshold. JAMA Surg. 2017;152(4): $360-8$.
30 Spaite DW, Hu C, Bobrow BJ, Chikani V, Barnhart B, Gaither JB, et al. The Effect of Combined Out-of-Hospital Hypotension and Hypoxia on Mortality in Major Traumatic Brain Injury. Ann Emerg Med. 2017;69(1):62-72.

31 Spaite DW, Bobrow BJ, Keim SM, Barnhart B, Chikani V, Gaither JB, et al. Association of Statewide Implementation of the Prehospital Traumatic Brain Injury Treatment Guidelines With Patient Survival Following Traumatic Brain Injury: The Excellence in Prehospital Injury Care (EPIC) Study. JAMA Surg. 2019; 154(7):e191152

32 Chang R, Folkerson LE, Sloan D, Tomasek JS, Kitagawa RS, Choi HA, et al. Early plasma transfusion is associated with improved survival after isolated traumatic brain injury in patients with multifocal intracranial hemorrhage. Surgery. 2017;161(2):538-45.

33 Jokar TO, Khalil M, Rhee P, Kulvatunyou N, Pandit V, O'Keeffe T, et al. Ratio-based Resuscitation in Trauma Patients with Traumatic Brain Injury: Is There a Similar Effect? Am Surg. 2016;82(3):271-7.

34 Meyer DE, Cotton BA, Fox EE, Stein D, Holcomb JB, Cohen $\mathrm{M}$, et al. A comparison of resuscitation intensity and critical administration threshold in predicting early mortality among bleeding patients: A multicenter validation in 680 major transfusion patients. J Trauma Acute Care Surg. 2018;85(4):691-6.

35 Blumberg N, Cholette JM, Pietropaoli AP, Phipps R, Spinelli SL, Eaton MP, et al. 0.9\% $\mathrm{NaCl}$ (Normal Saline) - Perhaps not so normal after all? Transfus Apher Sci. 2018;57(1):12731.

36 Yazer MH, Cap AP, Spinella PC. Raising the standards on whole blood. J Trauma Acute Care Surg. 2018;84(6S Suppl 1):S14-S7.

37 Yazer MH, Cap AP, Spinella PC, Alarcon L, Triulzi DJ. How do I implement a whole blood program for massively bleeding patients? Transfusion. 2018;58(3):622-8.

38 Giancarelli A, Birrer KL, Alban RF, Hobbs BP, Liu-DeRyke X. Hypocalcemia in trauma patients receiving massive transfusion. J Surg Res. 2016;202(1):182-7.

39 Webster S, Todd S, Redhead J, Wright C. Ionised calcium levels in major trauma patients who received blood in the Emergency Department. Emerg Med J. 2016;33(8):569-72.

40 MacKay EJ, Stubna MD, Holena DN, Reilly PM, Seamon MJ, Smith BP, et al. Abnormal Calcium Levels During Trauma Resuscitation Are Associated With Increased Mortality, Increased Blood Product Use, and Greater Hospital Resource Consumption: A Pilot Investigation. Anesth Analg. 2017;125(3): 895-901.

41 Pati S, Matijevic N, Doursout MF, Ko T, Cao Y, Deng X, et al. Protective effects of fresh frozen plasma on vascular endothelial permeability, coagulation, and resuscitation after hemorrhagic shock are time dependent and diminish between days 0 and 5 after thaw. J Trauma. 2010; 69(Suppl 1):S55-63.

42 Makris M, Greaves M, Phillips WS, Kitchen S, Rosendaal FR, Preston EF. Emergency oral anticoagulant reversal: the relative efficacy of infusions of fresh frozen plasma and clotting factor concentrate on correction of the coagulopathy. Thromb Haemost. 1997;77(3):477-80. 